\title{
SUPERFIELD EQUATIONS FOR SCALAR AND SPINOR SUPERFIELDS
}

\author{
(Presented by H. Keres)
}

General and linearized superfield equations for scalar and spinor superfields are treated. Some new equations are given. All the linear equations for chiral scalar superfield are physically equivalent. The concept of Klein-Gordon divisor for superfield equations is introduced.

\section{Introduction}

The concept of superfield has proved to be a useful instrument dealing with the supersymmetry. It allows to deal with the more compact and simpler expressions than operating in terms of component fields. On the other hand, the general superfield is a rather complicated object containing superfields with different superspins.

The irreducible representations of the super-Poincaré algebra are labelled by the mass and superspin [ [1]. In order to separate irreducible representations, the superfield equations of motion are needed. Some general principles of how to derive superfield equations were given in $\left[{ }^{2}\right]$. Using projection operators for a given superspin, it is possible to write down the Klein-Gordon equation for that superspin. Taking square roots of projection operators, these equations may be linearized. It appears that the procedure given in $\left[{ }^{2}\right]$ is not a general one. There are also equations which are not the square roots of projection operators. It means that the superfield equations of motion need further investigation to work out the general structure of superfield equations.

In this paper we deal with the superfield equations for scalar and spinor superfields. The general and linearized equations were written down, using the method given in $\left[{ }^{2}\right]$. In the case of scalar superfield it appears that all the linear equations for chiral superfield are physically equivalent. Similarly, as in the case of ordinary relativistic wave equations, it is useful to introduce the concept of Klein-Gordon divisor.

The notations we used are the same as in $\left[{ }^{3,4}\right]$, except for $\sigma^{\mu v}=$ $=\left[\gamma^{\mu}, \gamma^{v}\right] / 2$ and the usage of covariant and contravariant indices.

\section{General equation}

The general superfield with an external Lorentz index $k$ has a form

$$
\begin{gathered}
\Phi_{k}(x, \theta)=A_{k}(x)+\bar{\theta} \psi_{k}(x)+\frac{1}{4} \bar{\theta} \theta F_{k}(x)+\frac{1}{4} \bar{\theta} \gamma^{5} \theta G_{k}(x)+ \\
\left.+\frac{1}{4} \bar{\theta} i \gamma^{\mu} \gamma^{5} \theta A_{\mu k}(x)+\frac{1}{4} \bar{\theta} \bar{\theta} \bar{\theta} \chi_{k}(x)+\frac{1}{32} \overline{(\theta} \theta\right)^{2} D_{k}(x),
\end{gathered}
$$

where $\theta$ denotes four-component anticommuting Majorana spinor coor- 
dinate and $A_{k}(x), \psi_{k}(x), \ldots, D_{k}(x)$ are ordinary fields. In this paper wê deal with the scalar superfield $\Phi(x, \theta)$ and spinor superfield $\Psi_{\alpha}(x, \theta)$.

As it was mentioned above, the irreducible representations are classified by the mass $m$ and superspin $Y$. The general mass condition means that the components of a given superspin must satisfy the Klein-Gordon equation. That is obtained by the general equation

$$
\left(\square E^{Y}+m^{2}\right) \Phi_{k}=0,
$$

where $\square=\partial_{\mu} \partial^{\mu}, E^{Y}$ is the corresponding projection operator and $\Phi_{k}$ is the general superfield.

Acting on (2) with the projection operators $E^{\mathbf{Y}^{\prime}}$ and using $E^{\mathbf{Y}^{\mathbf{S}}} E^{\mathbf{Y}^{\prime}}=$ $=E^{Y} \delta_{Y Y^{\prime}}$, it is easy to verify that the superfield $\Phi_{k}^{Y}$ satisfies the KleinGordon equation, and $\Phi_{k}^{Y^{\prime}}=0$ if $Y^{\prime} \neq Y$.

Scalar superfield $\Phi(x, \theta)$. The general scalar superfield $\Phi(x, \theta)$ is decomposed as follows: $\Phi=\Phi_{+}+\Phi_{-}+\Phi_{1}$, where the irreducible superfields $\Phi_{+}, \Phi_{-}$and $\Phi_{1}$ have superspins $Y=0,0,1 / 2$, respectively $\left[{ }^{3,4}\right]$. The corresponding projection operators $E_{+}, E_{-}$and $E_{1}$ are $\left[{ }^{3,4}\right]$

$$
\begin{aligned}
& E_{ \pm}=-\frac{1}{8 \square}(\bar{D} D)^{2} \mp \frac{i}{4 \square} \partial_{\mu} \bar{D} i \gamma^{\mu} \gamma^{5} D, \\
& E_{1}=1+\frac{1}{4 \square}(\bar{D} D)^{2},
\end{aligned}
$$

where $D$ is the covariant spinor derivative. All the operators invariant under the supersymmetry transformations are constructed out of $\partial$ and $D$.

In the case of superspin $Y=1 / 2$, we obtain from (2) and (4) the Wess equation [ [2]

$$
\left(\square+\frac{1}{4}(\bar{D} D)^{2}+m^{2}\right) \Phi=0
$$

The analysis of the equation (5) in terms of component fields is quite troublesome, but, using the projection operators $E_{ \pm}, E_{1}$ or the qualities of spinor derivatives $D\left[{ }^{3}\right]$, it is easy to verify that the components of irreducible superfield $\Phi_{1}=E_{1} \Phi$ satisfy $\left(\square+m^{2}\right) \Phi_{1}=0$.

Using the projection operators $E_{+}$and $E_{-}(3)$, one can write down analogical equations for two superfields with $Y=0$. Here we write down only the equation for the whole superspin $Y=0$

$$
\left(-\frac{1}{4}(\bar{D} D)^{2}+m^{2}\right) \Phi=0
$$

which is the equation $\left(\square\left(E_{+}+E_{-}\right)+m^{2}\right) \Phi=0$.

S pinor superfield $\Psi(x, \theta)$. The general spinor superfield $\Psi_{\alpha}(x, \theta)$ with the bispinor index $\alpha$ has the following decomposition: $\Psi=\Psi_{+}+\Psi_{-}+$ $+\Psi_{1}^{0}+\Psi_{1}^{1}$. The superfields $\Psi_{+}$and $\Psi_{-}$correspond to the superspin $Y=1 / 2, \Psi_{1}^{0}$ to the superspin $Y=0$ and $\Psi_{1}^{1}$ to the superspin $Y=1$. The superspins $Y=1 / 2$ are projected out by the same projection operators $E_{+}$and $E_{-} . E_{1}$ projects out the superspins $Y=0$ and $Y=1 . Y=1$ is projected out by the operator $\left[{ }^{2,5}\right]$

$$
E_{1}^{1}=\frac{3}{4}\left(1+\frac{1}{4 \square}(\bar{D} D)^{2}\right)-\frac{1}{8 \square} ; \partial^{\mu} \sigma_{\mu \nu} \gamma^{5} \bar{D} i \gamma^{v} \gamma^{5} D
$$

and $Y=0$ by the operator $E_{1}^{0}=E_{1}-E_{1}^{1}$ 


$$
E_{1}^{0}=\frac{1}{4}\left(1+\frac{1}{4 \square}(\bar{D} D)^{2}\right)+\frac{1}{8 \square} \partial^{\mu} \sigma_{\mu \nu} \gamma^{5} \bar{D} i \gamma^{v} \gamma^{5} \bar{D} .
$$

Using (7) and (8), one can write down the Klein-Gordon equations for the superspins $Y=1$ and $Y=0$

$$
\begin{aligned}
& \left(\frac{3}{4}\left(\square+\frac{1}{4}(\bar{D} D)^{2}\right)-\frac{1}{8} \partial^{\mu} \sigma_{\mu \nu} \gamma^{5} \bar{D} i \gamma^{v} \gamma^{5} D+m^{2}\right) \Psi=0, \\
& \left(\frac{1}{4}\left(\square+\frac{1}{4}(\bar{D} D)^{2}\right)+\frac{1}{8} \partial^{\mu} \sigma_{\mu \nu} \gamma^{5} \bar{D} i \gamma^{v} \gamma^{5} D+m^{2}\right) \Psi=0 .
\end{aligned}
$$

Similarly, using the projection operators given in $\left[{ }^{5}\right]$, one can write down the equations for other superfields.

\section{Linearized equations}

The Klein-Gordon equations for superfields may be linearized to obtain the equations which give the mass condition and connect the component fields. This linearization procedure is in some sence analogical to the procedure of how we get the Dirac equation from the Klein-Gordon equation. Similarly, the linearized equation must contain at least two superfields with the same superspin $Y$. The linearized equations are constructed from the operators $\bar{D} D$ and $\bar{D} \gamma^{5} D$ in the case of scalar superfield $\Phi(x, \theta)$ and from $\partial_{\mu}, \bar{D} D, \bar{D} \gamma^{5} D, \bar{D} i \gamma^{v} \gamma^{5} D$ and $\gamma$-algebra in the case of the spinor superfield $\Psi(x, \theta)$.

$\mathrm{Scalar}$ superfield $\Phi(x, \theta)$. Since the linearized equation must contain two superspins $Y$, it is possible to linearize only the equation (6) for the whole superspin $Y=0$. The general linearized equation has a form

$$
\frac{1}{2}\left(\cos \xi \cdot \bar{D} D+\sin \xi \cdot \bar{D} \gamma^{5} D\right) \Phi=m \Phi,
$$

where $\xi$ is some real parameter. This equation is obtained as follows: we write (11) in the form $\pi \Phi=m \Phi$, where $\pi=\alpha \bar{D} D+\beta \bar{D} \gamma^{5} D$, and demand in the following $\left[{ }^{2}\right]$ that $\pi^{2}=-\square\left(E_{+}+E_{-}\right)$or $\pi^{2}=(\bar{D} D)^{2} / 4$.

In $\left[{ }^{3}\right]$ the following linearized equation was delt with: $\bar{D} D \Phi=2 m \Phi$. It corresponds to the parameter $\xi=0$. If we write down (11) in terms of component fields, it is possible to verify that by a proper renormalization of the field components the equations with different $\xi$ are equivalent.

To conclude this section, we write down (11) in terms of irreducible components $\Phi_{+}, \Phi_{-}$and $\Phi_{1}$

$$
\begin{aligned}
\frac{1}{2}\left(\cos \xi \cdot \bar{D} D+\sin \xi \cdot \bar{D} \gamma^{5} D\right) \Phi_{-} & =m \Phi_{+}, \\
\frac{1}{2}\left(\cos \xi \cdot \bar{D} D+\sin \xi \cdot \bar{D} \gamma^{5} D\right) \Phi_{+} & =m \Phi_{-}, \\
0 & =m \Phi_{1} .
\end{aligned}
$$

Here we used the relations $E_{ \pm} \bar{D} D=\bar{D} D E_{\mp}, E_{ \pm} \bar{D} \gamma^{5} D=\bar{D} \gamma^{5} D E_{\mp}, E_{1} \bar{D} D=0$, and $E_{1} \bar{D} \gamma^{5} D=0$.

Spinor superfield $\Psi(x, \theta)$. In the case of the superspin $Y=1$, the general linearized equation (9) has a form $\left[{ }^{2}\right]$ 


$$
\begin{gathered}
\frac{1}{8}\left\{\left(\cos \xi+\gamma^{5} \sin \xi\right)\left(6 i \hat{\partial}-i \gamma_{v} \gamma^{5} \bar{D} i \gamma^{v} \gamma^{5} D\right)+\right. \\
\left.+\cos \eta\left(\bar{D} D+3 \gamma^{5} \bar{D} \gamma^{5} D\right)+\sin \eta \gamma^{5}\left(3 \bar{D} D+\gamma^{5} \bar{D} \gamma^{5} D\right)\right\} \Psi=m \Psi,
\end{gathered}
$$

where $\xi$ and $\eta$ are arbitrary real parameters.

In the case of the superspin $Y=0$, we analogically get the linearized equation (10)

$$
\begin{aligned}
& \frac{1}{8}\left\{\left(\cos \xi+\gamma^{5} \sin \xi\right)\left(2 i \hat{\partial}+i \gamma_{\nu} \gamma^{5} \bar{D} i \gamma^{v} \gamma^{5} D\right)+.\right. \\
& \left.+\left(\cos \eta+\gamma^{5} \sin \eta\right)\left(\bar{D} D-\gamma^{5} \bar{D} \gamma^{5} D\right)\right\} \Psi=m \Psi .
\end{aligned}
$$

The linearization of the whole superspin $Y=1 / 2$ gets the following equation -

$$
\left\{a \sin \xi \frac{1 \pm i \gamma^{5}}{2}\left(6 i \hat{\partial}+5 i \gamma v \gamma^{5} \bar{D} i \gamma^{v} \gamma^{5} D\right)+\right.
$$

$\left.+\frac{1}{8}\left[\cos \eta\left(5 \bar{D} D-3 \gamma^{5} \bar{D} \gamma^{5} D\right)-\sin \eta \cdot \gamma^{5}\left(3 \bar{D} D-5 \gamma^{5} \bar{D} \gamma^{5} D\right)\right]\right\} \Psi=m \Psi$,

where $a$ is also a free parameter.

The equations (13)-(15), which are derived using the prescription of paper $\left[{ }^{2}\right]$, have a common quality: they are written in the form $\pi \Psi=$ $=m \Psi$, where $\pi^{2}=-\square E^{Y}$. Therefore $\pi$ satisfies the minimal equation

$$
\pi^{4}=-\square \pi^{2} \text {. }
$$

Similarly as in the case of ordinary relativistic wave equations, we define the Klein-Gordon divisor $d$ by the relation (see, for example $\left.{ }^{6}\right]$ )

$$
d(\pi-m)=-\left(\square+m^{2}\right) \text {. }
$$

From (16) $d$ has a form

$$
d=(\pi+m)\left[1+\frac{1}{m^{2}}\left(\pi^{2}+\square\right)\right] .
$$

The inverse operator $(\pi-m)^{-1}$ which defines the Green function, is easily found from (17) and (18)

$$
\frac{1}{\pi-m}=-\frac{d}{\square+m^{2}} \equiv-\frac{\pi+m}{\square+m^{2}}\left[1+\frac{1}{m^{2}}\left(\pi^{2}+\square\right)\right] .
$$

\section{Chiral superfields}

Using the chiral superfields $\Phi_{+}, \Phi_{-}$or $\Psi_{+}, \Psi_{-}$, it is possible to obtain separate equations for superspins $Y=0$ or $Y=1 / 2$ which differ from the equations described in the previous sections.

Scalar superfields $\Phi_{+}, \Phi_{-}$. One of the possible equations is the equation (12) where we may leave out $\Phi_{1}$. But it is possible to obtain a new equation which differs from (12). The superspin $Y=0$ is also described by an equation

$$
\left(\alpha \bar{D} D+\beta \bar{D} \gamma^{5} D\right) \Phi-=m \Phi_{+}, \quad\left(\gamma \bar{D} D+\delta \bar{D} \gamma^{5} D\right) \Phi_{+}=m \Phi_{-},
$$

where $\alpha, \beta, \gamma$ and $\delta$ satisfy

$$
\alpha \gamma+\beta \delta=1 / 8, \quad \alpha \delta-\beta \gamma=i / 8 .
$$


Denoting

$$
\pi=\left|\begin{array}{cc}
0 & \alpha \bar{D} D+\beta \bar{D} \gamma^{5} D \\
\gamma \bar{D} D+\delta \bar{D} \gamma^{5} D & 0
\end{array}\right|, \quad \Phi=\left|\begin{array}{c}
\Phi_{+} \\
\Phi_{-}
\end{array}\right|
$$

(20) has a form $\pi \Phi=m \Phi$, where

$$
\pi^{2}=-\square\left|\begin{array}{cc}
E_{+} & 0 \\
0 & E_{-}
\end{array}\right| \text {. }
$$

In the case of parameters $\alpha=\gamma=1 / 4, \delta=-\beta=i / 4$, the equation (20) is connected with the following decomposition of projection operators $E_{ \pm}$

$$
E_{ \pm}=-\frac{1}{\square} \Gamma_{\mp} \Gamma_{ \pm}
$$

where

$$
\Gamma_{ \pm}=\bar{D} \frac{1 \pm i \gamma^{5}}{4} D
$$

Now $(20)$ has a form

$$
\Gamma_{-} \Phi_{-}=m \Phi_{+}, \quad \Gamma_{+} \Phi_{+}=m \Phi_{-} .
$$

In terms of independent component fields of chiral superfields $\Phi_{ \pm}$; $A_{ \pm}(x), \psi_{ \pm}(x)$ and $F_{ \pm}(x)(26)$ gives the same equations as (12) in the case of $\xi=0$

$$
-F_{\mp}=m A_{ \pm}, \quad i \hat{\partial}_{\psi_{\mp}}=m \psi_{ \pm}, \quad \square A_{\mp}=m F_{ \pm} .
$$

In the case of $\Phi_{-}=\Phi_{+}^{*}$, it is the Wess-Zumino equation for a free chiral scalar superfield $\left[{ }^{7}\right]$.

In the case of other parameters $\alpha, \beta, \gamma$ and $\delta$ satisfying (21), we obtain the same equations (2) by the renormalization of field components. Therefore, in the ftee field level, all the equations for chiral scalar superfield are physically equivalent and give the component equations (27).

For chiral superfield equations Klein-Gordon divisor $d$ is written in the form

$$
d=\pi+m .
$$

Now $E_{ \pm}$act as identity operators, and therefore $\pi^{2}=-\square$.

Spinor superfields $\Psi_{+}, \Psi_{-}$. It is possible to give the same equations (12) or $(20)$ for chiral spinor superfields $\Psi_{+}$and $\Psi_{-}$. These equations are not physically interesting, since the spinor component fields satisfy the Klein-Gordon equation.

The equation for $\Psi_{+}$and $\Psi_{-}$which, in principle, differs from the linearized equations given above, was given in $\left[{ }^{3}\right]$

$$
(i \hat{\partial}-2 m) \Psi_{+}+\frac{1}{2} \bar{D} D \Psi_{-}=0, \quad(i \hat{\partial}-2 m) \Psi_{-}+\frac{1}{2} \bar{D} D \Psi_{+}=0 .
$$

This equation can be somewhat generalized. Denoting

$$
\pi=\frac{1}{4}\left|\begin{array}{cc}
2 i \hat{\partial} & \alpha \bar{D} D \\
\beta \bar{D} D & 2 i \hat{\partial}
\end{array}\right|, \quad \Psi=\left|\begin{array}{c}
\Psi_{+} \\
\Psi_{-}
\end{array}\right|,
$$

we write $(29)$ in a standard form

$$
\pi \Psi=m \Psi:
$$


Now $\pi^{2} \neq-\square E^{Y}$, but $\pi$ satisfies

$$
\pi^{3}=-\square \pi
$$

if the coefficients $\alpha$ and $\beta$ satisfy $\alpha \beta=1$ :

The Klein-Gordon divisor $d$ has a form

$$
d=\pi+m+\frac{1}{m}\left(\pi^{2}+\square\right) .
$$

As we have mentioned, this equation in principle differs from the linearized equations derived by the method of the paper $\left[{ }^{2}\right]$. Since $\pi^{2} \neq$ $\neq-\square E^{Y}$, equation (31) is not a square root of the projection operator. It is also interesting to note that the tensor component fields in $\Psi$ satisfy the Kemmer-Duffin equation $\left[{ }^{8}\right]$.

\section{Conclusions}

In this paper we dealt with the general and linearized superfield equations for scalar and spinor superfields. The general mass condition for a given superspin means that the corresponding field components satisfy the Klein-Gordon equation. The latter is achieved by making use of the respective projection operators. In the superfield case, the operators $\square E^{Y}$ are all local operators, and therefore it is always possible to use the general equation (2).

The linearized equations are constructed out of operators $\partial_{\mu} ; \bar{D} D$, $\bar{D} \gamma^{5} D, \bar{D} i \gamma^{v} \gamma^{5} D$ and some matrix algebra, depending on the choice of external Lorentz index $k$ in (1). In the case of spinor superfield, one must use the Dirac $\gamma$-algebra. One of the possible classes of linear equations are obtained by the procedure proposed in $\left[{ }^{2}\right]$. According to this procedure, one must take the square root of operator $\square E^{Y}$. There are also equations squares of which are not equal to $\square E^{Y}$; thus, it should be clear that other possible classes of superfield equations exist as well. Since the superfield equations are not yet investigated enough, it is interesting to clarify the structure of both existing and new superfield equations.

As it has been pointed out in $\left[{ }^{2}\right]$, there is some analogy between the construction of ordinary relativistic wave equations and superfield equations. It seems that the concept of Klein-Gordon divisor is also useful in the theory of superfield equations.

\section{REFERENCES}

1. Salam, A., Strathdee, J. Nucl. Phys., B80, № 3, 499-505 (1974).

2. Ogievetsky, V. I., Sokatchev, E. J. Phys. A: Math. Gen., 10, № 11, 2021-2030 (1977),

3. Salam, A., Strathdee, J. Phys. Rev., Di1, № 6, 1521-1535 (1975).

4. Salam, A., Strathdee, J. Fortschr. Phys., 26, № 2, 57-142 (1978).

5. Sokatchev, E. Nucl. Phys., B99, № 1, 96-108 (1975).

6. Умедзава Х. Квантовая теория поля. М., ИЛ, 1958.

7. Wess, J., Zumino, B. Phys. Lett., B49, № 1, 52-54 (1974).

8. Adjei, S. A., Akyeampong, D. A. Nuovo Cim., A26, № 1, 84-93 (1975). 


\section{SUPERVĂLJAVORRANDID SKALAAR- JA SPIINORSUPERVÄLJALE}

Artikkel käsitleb üldisi ja lineariseeritud superväljavõrrandeid skalaar- ja spiinorsuperväljale. On antud mõned uued võrrandid. Kõik lineaarsed kiraalse skalaarsupervälja võrrandid on füüsikaliselt ekvivalentsed. Superväljavõrrandite tarvis on sisse toodud Klein-Gordoni jagaja mõiste.

\section{Р.-К. ЛОИДЕ, П. СУУРВАРИК}

\section{СУПЕРПОЛЕВЫЕ УРАВНЕНИЯ ДЛЯ СКАЛЯРНОГО И СПИНОРНОГО СУПЕРПОЛЕЙ}

Рассматриваются общие и линеаризованные уравнения для скалярного и спинорного суперполей. Дается несколько новых уравнений. Все линейные уравнения кирального скалярного суперполя физически эквивалентны. Для суперполевых уравнений дается понятие делителя Клейна-Гордона. 